Kragujevac Journal of Mathematics

Volume 41(2) (2017), Pages 259-278.

\title{
CURVATURE PROPERTIES OF SOME 4-DIMENSIONAL SEMI-RIEMANNIAN METRICS
}

\author{
A. A. SHAIKH ${ }^{1}$ AND Y. MATSUYAMA ${ }^{2}$ \\ Dedicated to the memory of Professor Mileva Prvanović
}

\begin{abstract}
The objective of the present paper is to investigate the geometric structures admitting by a metric which is physically relevant as well as significant. The curvature properties of the metric investigated in the paper are also significant geometrically as the metric admits several kinds of pseudosymmetric type conditions among which some of them are new in literature.
\end{abstract}

\section{INTRODUCTION}

Let $M$, dimension $M=n \geq 3$, be a connected semi-Riemannian smooth manifold endowed with a semi-Riemannian metric $g$ of signature $(s, n-s), 0 \leq s \leq n$. If $s=0$ or $s=n$ then $M$ is a Riemannian manifold, and if $s=1$ or $s=n-1$, then $M$ is a Lorentzian manifold. The nature of a semi-Riemannian manifold is determined by its curvature tensors, viz., the Riemann curvature tensor $R$, Ricci curvature $S$ and scalar curvature $r$. The notion of three curvatures are equivalent for any two dimensional semi-Riemannian manifold but not for $n \geq 3$. Hence throughout the paper we will consider a smooth connected semi-Riemannian manifold of dimension $n \geq 3$.

The Ricci curvature is the trace of the Riemann curvature and the scalar curvature is the trace of the Ricci curvature. Hence to investigate the nature of a manifold one should determine its Riemann curvature tensor. The metric tensor $g$ determines $R$ by means of second order partial derivatives with respect to the coordinate functions. Thus ultimately the metric tensor $g$ determines the geometry of the manifold which

Key words and phrases. Recurrent, pseudosymmetric, Ricci generalized pseudosymmetric manifold, Weyl conformal curvature tensor, projective tensor, conharmonic tensor, concircular tensor, pseudosymmetric type condition.

2010 Mathematics Subject Classification. Primary: 53B20. Secondary: 53C15, 53C25, 53C35.

Received: April 25, 2015.

Accepted: September 11, 2016. 
is non-flat. In general relativity a spacetime is a connected 4-dimensional Lorentzian manifold. General relativity is described by Einstein field equations which is the set of equations connecting the geometrical properties of the spacetime with the distribution of gravitating matter contained in the spacetime. The energy momentum tensor contains all the information about the matter contained in the spacetime. Thus Einstein field equations implies that a second rank tensor which describes the geometry of the spacetime is equal to a second rank tensor which describes the physics of the spacetime. Therefore Einstein field equations are fundamental in the construction of a cosmological model which implies that the matter gives rise to the geometry of the spacetime and conversely the motion of matter is determined by the metric tensor of the non-flat space. In general relativity there are many metrics which describes various cosmological models and each of the metric are physically relevant and significant and their physical properties are well known due to the information of a specific cosmological model. However, this physically significant metrics may have carried out many geometric properties which are not well studied. The geometric structures are obtained by imposing the first and higher order covariant derivatives on the Riemann curvature tensor and also other curvature tensors which arise as an invariant of specific transformation on a manifold, for example, the conformal, concircular, conharmonic, projective curvature tensor etc. However, all these invariant curvature tensors are mainly the linear combination of the tensors formed by $g, R, S$ and $r$. Thus ultimately the metric tensor $g$ plays the crucial role in the study of differential geometry for the characterization and classification of different geometric structures. However, in differential geometry a specific geometric structure can be classified locally which ensures the form of metric of that structure and hence all the geometric information of the space can be determined. But there are many geometric structures whose local classification is either difficult or cumbersome and yet known to be impossible. In all those cases the existence of such structures is guaranteed by means of specific metrics which are the trend of modern mathematics as abstraction, generalization or extension, existence and then application. In the literature of differential geometry there are many geometric structures which are determined by means of its curvature tensor with first order or higher order covariant derivatives, for example, the local symmetry, semisymmetry, recurrency, pseudosymmetry, weakly symmetry etc. The moto of this paper is unconventional because we will consider a physically relevant metric and then we would like to determine its curvature properties and verify which geometric structures are admitted by this metric and which are not admitted by this metric. Then it will be a nice approach to conclude that this metric will be a model of such geometric structures which are admitting by this metric. For this purpose let us consider the metric defined on a non-empty open connected subset of $\mathbb{R}^{4}$ as follows:

$$
\begin{aligned}
d s^{2} & =g_{i j} d x^{i} d x^{j} \\
& =(1+2 p)\left[\left(d x^{1}\right)^{2}+\left(d x^{2}\right)^{2}+\left(d x^{3}\right)^{2}\right]+\left(d x^{4}\right)^{2}, \quad i, j=1,2,3,4
\end{aligned}
$$


where $p=\frac{e^{x^{1}}}{\rho^{2}}$ and $\rho$ is a non-zero constant; $x^{1}, \ldots, x^{4}$ are the standard coordinates of $\mathbb{R}^{4}$. We note that such type of metric was first appeared in [89] and latter used by many authors (see $[15,16,100])$ to prove the existence of various curvature restricted geometric structures.

This paper is organized as follows. Section 2 deals with rudiments of various geometric structures which are essential to study the curvature properties of the above described metric. Section 3 is mainly concerned with the various components of different curvature tensors to investigate the geometric structures admitting by this metric. The last section is devoted to the conclusion as theorems (see Theorems 4.1 and 4.2) and remarks.

\section{Preliminaries}

Let $\nabla$ be the Levi-Civita connection of $M$. In 1926 Cartan introduced the notion of locally symmetric manifolds with full classification $[7,8]$. $M$ is called locally symmetric due to Cartan if $\nabla R=0$, which is equivalent to the fact that the local geodesic symmetry at each point of $M$ is an isometry. During the last eight decades the notion of locally symmetric spaces have been generalized by many authors in different ways and several steps such as $\kappa$-space by Ruse [73-75] (which is called recurrent space by Walker in 1950 [114]), conformally recurrent manifolds by Adati and Miyazawa [2], projectively recurrent manifolds by Adati and Miyazawa [3], 2-recurrent manifolds by Lichnerowicz [57], generalized recurrent manifolds by Dubey [47], quasi-generalized recurrent manifolds by Shaikh and Roy [101], hyper generalized recurrent manifolds by Shaikh and Patra [100], weakly generalized recurrent manifolds by Shaikh and Roy [102], semisymmetric manifolds by Cartan [9] (which were classified by Szabó [107-109], in the Riemannian case), pseudosymmetric manifolds by Deszcz [23,35, 83], pseudosymmetric manifolds by Chaki [10], weakly symmetric manifolds by Selberg [78], weakly symmetric manifolds by Tamássy and Binh [110]. It may be mentioned that the notion of weakly symmetric manifold by Selberg is different from that by Tamássy and Binh [83], and pseudosymmetric manifold by Chaki is also different from pseudosymmetric manifold by Deszcz [83]. The manifold $M$ is said to be Ricci symmetric if $\nabla S=0$ (see, e.g., [69]). The notion of Ricci symmetry was also weakend by various ways such as Ricci recurrent by Patterson [70], Ricci semisymmetric by Okumara [69], Roter [72], Tanno [112], Ryan [76], Matsuyama [66], Mirzoyan [68], Abdalla and Dillen [1] and others, Ricci pseudosymmetric by Deszcz [21], pseudo Ricci symmetric by Chaki [11], weakly Ricci symmetric by Tamássy and Binh [111].

To find an exact solution of Einstein field equation, initially, a geometric structure arose in the space which is called an Einstein space. A connected semi-Riemannian manifold $(M, g), n \geq 3$, is called Einstein if its Ricci tensor $S$ of type $(0,2)$ is a constant multiple of the metric tensor $g$ such that $S=\frac{r}{n} g$ [4]. As a direct and algebraic generalization of an Einstein manifold, the notion of quasi-Einstein manifold 
arose during the study of exact solutions of the Einstein's field equation (FriedmannLemaître-Robertson-Walker spacetimes; see, e.g., Section 6 of [51] and [56]) as well as during the investigation of quasi-umbilical hypersurfaces by Cartan [6] and Schouten [77]. Let $U_{S}=\left\{x \in M:\left(S-\frac{r}{n} g\right)_{x} \neq 0\right\}$. Then the manifold is said to be quasiEinstein $[14,19,25,28,39,40,43,49,56,69,88,94,105]$ if on $U_{S} \subset M$, we have

$$
S-\alpha g=\beta A \otimes A,
$$

where $A$ is an 1 -form on $U_{S}$ and $\alpha, \beta$ are some functions on $U_{S}$ and $\otimes$ is the tensor product. It is clear that the 1 -form $A$ as well as the function $\beta$ are non-zero at every point on $U_{S}$. The manifold is said to be a 2 -quasi-Einstein $[29,31,33]$ manifold if $\operatorname{rank}(S-\alpha g) \leq 2$ and $\operatorname{rank}(S-\alpha g)=2$ on some open non-empty subset of $U_{S}$, where $\alpha$ is some function on $U_{S}$.

Again as in an Einstein manifold the Ricci tensor $S$ is proportional to the metric tensor $g$, the Ricci parallel condition in the manifold i.e., $\nabla S=0$ is a generalized structure of an Einstein manifold and also manifold of constant scalar curvature is a generalization of Ricci parallel. In 1978 Gray [50] obtained two classes $\mathcal{A}, \mathcal{B}$ of Riemannian manifolds which lie between the class of Ricci symmetric manifolds and the manifolds of constant scalar curvature. The class $\mathcal{A}$ (resp. $\mathcal{B}$ ) is the class of Riemannian manifolds whose Ricci tensor is cyclic parallel (resp. Codazzi tensor). Every Ricci symmetric manifold is of class $\mathcal{B}$ but not conversely. We note that every manifold of constant curvature and hence an Einstein manifold is of class $\mathcal{A}$ as well as $\mathcal{B}$. The existence of both the classes is given in [82]. In a semi-Riemannian manifold $(M, g), n \geq 3$, the Ricci tensor $S$ is said to be a Codazzi tensor $[48,106]$ (resp. cyclic Ricci parallel [50]), if it satisfies

$$
\begin{gathered}
\left(\nabla_{X_{1}} S\right)\left(X_{2}, X_{3}\right)=\left(\nabla_{X_{2}} S\right)\left(X_{1}, X_{3}\right) \\
\text { (resp. } \left.\quad\left(\nabla_{X_{1}} S\right)\left(X_{2}, X_{3}\right)+\left(\nabla_{X_{2}} S\right)\left(X_{3}, X_{1}\right)+\left(\nabla_{X_{3}} S\right)\left(X_{1}, X_{2}\right)=0\right),
\end{gathered}
$$

for all vector fields $X_{1}, X_{2}, X_{3} \in \chi(M)$, where $\chi(M)$ is the Lie algebra of all smooth vector fields on $M$.

As a generalization of symmetry there arises recurrent structures such as recurrent and Ricci recurrent manifold. Let $U_{L}=\{x \in M: R \neq 0$ at $x\}$ and $U_{N}=\{x \in M$ : $S \neq 0$ at $x\}$. A semi-Riemannian manifold $(M, g), n \geq 3$, is said to be recurrent [114] if on $U_{L} \subset M$ the condition $\nabla R=A \otimes R$ holds and Ricci recurrent [70] if on $U_{N} \subset M$ the condition $\nabla S=A \otimes S$ holds, where $A$ is a non-zero 1-form.

Again to generalize the notion of recurrent manifold in [96,98, 100-103] (see also [80]) the following four curvature conditions were introduced

$$
\begin{aligned}
\nabla R & =A \otimes R+B \otimes(G+g \wedge H), \\
\nabla R & =A \otimes R+B \otimes(S \wedge g), \\
\nabla R & =A \otimes R+B \otimes \frac{1}{2}(S \wedge S), \\
\text { and } \nabla R & =A \otimes R+B_{1} \otimes(S \wedge S)+B_{2} \otimes(g \wedge S)+B_{3} \otimes(g \wedge g),
\end{aligned}
$$


for some non-zero 1-forms $A, B, B_{1}, B_{2}, B_{3}$ such that $A(X)=g(\sigma, X), B(X)=$ $g(\rho, X), B_{1}(X)=g\left(\rho_{1}, X\right), B_{2}(X)=g\left(\rho_{2}, X\right)$ and $B_{3}(X)=g\left(\rho_{3}, X\right), H=\eta \otimes \eta, \eta$ being a non-zero 1-form, and the Kulkarni-Nomizu product $E \wedge F$ of two (0,2)-tensors $E$ and $F$ is defined by (see e.g. $[27,49,55])$

$$
\begin{aligned}
(E \wedge F)\left(X_{1}, X_{2}, X_{3}, X_{4}\right)= & E\left(X_{1}, X_{4}\right) F\left(X_{2}, X_{3}\right)+E\left(X_{2}, X_{3}\right) F\left(X_{1}, X_{4}\right) \\
& -E\left(X_{1}, X_{3}\right) F\left(X_{2}, X_{4}\right)-E\left(X_{2}, X_{4}\right) F\left(X_{1}, X_{3}\right),
\end{aligned}
$$

$X_{1}, X_{2}, X_{3}, X_{4} \in \chi(M)$. A non-flat semi-Riemannian manifold $(M, g), n \geq 3$, satisfying the respective conditions in $\left\{x \in M:(R)_{x} \neq 0\right.$ and $\left.(G+g \wedge H)_{x} \neq 0\right\}$, $\left\{x \in M:(R)_{x} \neq 0\right.$ and $\left.(g \wedge S)_{x} \neq 0\right\},\left\{x \in M:(R)_{x} \neq 0\right.$ and $\left.(S \wedge S)_{x} \neq 0\right\}$ and $\left\{x \in M:(R)_{x} \neq 0\right.$ and any one of $S \wedge S, g \wedge S$ is non-zero at x $\}$ are respectively called quasi-generalized recurrent manifold [101] (briefly, $Q G K_{n}$ ), hyper generalized recurrent manifold [100] (briefly, $H G K_{n}$ ), weakly generalized recurrent manifold [102] (briefly, $W G K_{n}$ ) and super generalized recurrent manifold [96,98,103] (briefly, $S G K_{n}$ ). Obviously, every $H G K_{n}$ and $W G K_{n}$ is a $S G K_{n}$. We note that for $\alpha=\beta$ a quasiEinstein manifold is $W G K_{n}$ if and only if it is $Q G K_{n}$. Again, we also note that for $2 \alpha=\beta$ a quasi-Einstein manifold is $H G K_{n}$ if and only if it is $Q G K_{n}$.

A semi-Riemannian manifold $(M, g), n \geq 3$, is said to be weakly symmetric by Tamássy and Binh [110] if on $U_{L} \subset M$ the following condition holds:

$$
\begin{aligned}
\left(\nabla_{X} R\right)\left(X_{1}, X_{2}, X_{3}, X_{4}\right)= & A(X) R\left(X_{1}, X_{2}, X_{3}, X_{4}\right) \\
& +B\left(X_{1}\right) R\left(X, X_{2}, X_{3}, X_{4}\right)+B\left(X_{2}\right) R\left(X_{1}, X, X_{3}, X_{4}\right) \\
& +D\left(X_{3}\right) R\left(X_{1}, X_{2}, X, X_{4}\right)+D\left(X_{4}\right) R\left(X_{1}, X_{2}, X_{3}, X\right),
\end{aligned}
$$

for all vector fields $X, X_{i} \in \chi(M)(i=1,2,3,4)$ and some 1-forms $A, B, D$ on $M$. Weakly symmetric manifolds were also studied in [52, 81, 84-87, 90-93, 95, 99, 104].

The semi-Riemannian manifold $(M, g), n \geq 3$, is called weakly Ricci symmetric [111] if on $U_{N}$

$$
\left(\nabla_{X} S\right)\left(X_{1}, X_{2}\right)=A(X) S\left(X_{1}, X_{2}\right)+B\left(X_{1}\right) S\left(X, X_{2}\right)+D\left(X_{2}\right) S\left(X_{1}, X\right),
$$

holds for some 1-forms $A, B, D$ (not simultaneously zero), where $X, X_{1}, X_{2} \in \chi(M)$.

Investigating conformally flat Riemannian manifolds of class one, that is manifolds characterized by the property that at least $n-1$ principal normal curvatures (i.e., the eigenvalues of the second fundamental form) are equal to one another, Sen and Chaki [79] found that if the remaining one is zero, then the curvature tensor satisfies

$$
\left(\nabla_{X} R\right)\left(X_{1}, X_{2}, X_{3}, X_{4}\right)=2 A(X) R\left(X_{1}, X_{2}, X_{3}, X_{4}\right)
$$

$$
\begin{aligned}
& +A\left(X_{1}\right) R\left(X, X_{2}, X_{3}, X_{4}\right)+A\left(X_{2}\right) R\left(X_{1}, X, X_{3}, X_{4}\right) \\
& +A\left(X_{3}\right) R\left(X_{1}, X_{2}, X, X_{4}\right)+A\left(X_{4}\right) R\left(X_{1}, X_{2}, X_{3}, X\right),
\end{aligned}
$$

for some non-zero 1-form $A$, where $X, X_{1}, X_{2}, X_{3}, X_{4} \in \chi(M)$. However, Riemannian manifolds with condition (2.2) imposed on the curvature tensor were examined by Chaki [10] and Chaki and De [12], and Chaki called such manifolds pseudosymmetric. We mention that conformally flat semi-Riemannian manifolds of class one are also 
pseudosymmetric by Deszcz. This is an immediate consequence of Theorem 4.1 of [44] and Corollary 1 of [45].

Again, the manifold $(M, g), n \geq 3$, is said to be pseudo Ricci symmetric [11] if on $U_{N} \subset M$ its Ricci tensor $S$ is not identically zero and satisfies following:

$$
\left(\nabla_{X} S\right)\left(X_{1}, X_{2}\right)=2 A(X) S\left(X_{1}, X_{2}\right)+A\left(X_{1}\right) S\left(X, X_{2}\right)+A\left(X_{2}\right) S\left(X_{1}, X\right),
$$

for some non-zero 1-form $A$, where $X, X_{1}, X_{2} \in \chi(M)$.

We refer to [5] and [67] for a presentation of Professor Mileva Prvanović's contribution to Differential Geometry, and in particular to geometry of recurrent, generalized recurrent, conformally flat, conformally symmetric, conformally recurrent, conformally quasi-recurrent and weakly symmetric manifolds.

All the structures discussed above are equipped with the condition involving the first order covariant derivatives. Now we will discuss some geometric structures obtained by second order covariant derivatives which are either generalization or extension of some well known geometric structures. For this purpose we first discuss some notations as follows.

The endomorphisms $X \wedge_{E} Y$ and $\mathcal{R}(X, Y)$ are, respectively, given by [19,27,49]

$$
\left(X \wedge_{E} Y\right) X_{1}=E\left(Y, X_{1}\right) X-E\left(X, X_{1}\right) Y \text { and } \mathcal{R}(X, Y) X_{1}=\left[\nabla_{X}, \nabla_{Y}\right] X_{1}-\nabla_{[X, Y]} X_{1}
$$

where $E$ is a $(0,2)$-tensor on $M$ and $X, Y, X_{1} \in \chi(M)$.

It is well known that the conformal transformation is an angle preserving mapping, the projective transformation is a geodesic preserving mapping whereas concircular transformation is the geodesic circle preserving mapping and conharmonic transformation is a harmonic function preserving mapping. Now we define the Gaussian curvature tensor $G$, the Riemann-Christoffel curvature tensor $R$, the Weyl conformal curvature tensor $C$, the projective curvature tensor $P$, the concircular curvature tensor $K$ and the conharmonic curvature tensor $Z$ of $(M, g)$, respectively, by $[19,27,49,53,115]$

$$
\begin{aligned}
G\left(X_{1}, X_{2}, X_{3}, X_{4}\right)= & g\left(\left(X_{1} \wedge g, X_{2}\right) X_{3}, X_{4}\right), \\
R\left(X_{1}, X_{2}, X_{3}, X_{4}\right)= & g\left(\mathcal{R}\left(X_{1}, X_{2}\right) X_{3}, X_{4}\right), \\
C\left(X_{1}, X_{2}, X_{3}, X_{4}\right)= & R\left(X_{1}, X_{2}, X_{3}, X_{4}\right)-\frac{1}{n-2}(g \wedge S)\left(X_{1}, X_{2}, X_{3}, X_{4}\right) \\
& +\frac{r}{(n-2)(n-1)} G\left(X_{1}, X_{2}, X_{3}, X_{4}\right), \\
P\left(X_{1}, X_{2}, X_{3}, X_{4}\right)= & R\left(X_{1}, X_{2}, X_{3}, X_{4}\right) \\
& -\frac{1}{n-1}\left[g\left(X_{1}, X_{4}\right) S\left(X_{2}, X_{3}\right)-g\left(X_{2}, X_{4}\right) S\left(X_{1}, X_{3}\right)\right], \\
K\left(X_{1}, X_{2}, X_{3}, X_{4}\right)= & R\left(X_{1}, X_{2}, X_{3}, X_{4}\right)-\frac{r}{n(n-1)} G\left(X_{1}, X_{2}, X_{3}, X_{4}\right), \\
Z\left(X_{1}, X_{2}, X_{3}, X_{4}\right)= & R\left(X_{1}, X_{2}, X_{3}, X_{4}\right)-\frac{1}{n-2}(g \wedge S)\left(X_{1}, X_{2}, X_{3}, X_{4}\right) .
\end{aligned}
$$


For an $(0, k)$-tensor $T, k \geq 1$ and a symmetric $(0,2)$-tensor $E$ we define the $(0, k)$ tensor $E \cdot T$ and the $(0, k+2)$-tensors $R \cdot T, C \cdot T$ and $Q(E, T)$ by $[19,27,34,49]$

$$
\begin{aligned}
(E \cdot T)\left(X_{1}, \cdots, X_{k}\right)= & -T\left(\mathcal{E} X_{1}, X_{2}, \cdots, X_{k}\right)-\cdots-T\left(X_{1}, X_{2}, \cdots, \mathcal{E} X_{k}\right), \\
(R \cdot T)\left(X_{1}, \cdots, X_{k} ; X, Y\right)= & (\mathcal{R}(X, Y) \cdot T)\left(X_{1}, \cdots, X_{k}\right) \\
= & -T\left(\mathcal{R}(X, Y) X_{1}, X_{2}, \cdots, X_{k}\right)-\cdots \\
& -T\left(X_{1}, \cdots, X_{k-1}, \mathcal{R}(X, Y) X_{k}\right) \\
(C \cdot T)\left(X_{1}, \cdots, X_{k} ; X, Y\right)= & (\mathcal{C}(X, Y) \cdot T)\left(X_{1}, \cdots, X_{k}\right) \\
= & -T\left(\mathcal{C}(X, Y) X_{1}, X_{2}, \cdots, X_{k}\right)-\cdots \\
& -T\left(X_{1}, \cdots, X_{k-1}, \mathcal{C}(X, Y) X_{k}\right), \\
Q(E, T)\left(X_{1}, \cdots, X_{k} ; X, Y\right)= & \left.\left(X \wedge_{E} Y\right) \cdot T\right)\left(X_{1}, \cdots, X_{k}\right) \\
= & -T\left(\left(X \wedge_{E} Y\right) X_{1}, X_{2}, \cdots, X_{k}\right)-\cdots \\
& -T\left(X_{1}, \cdots, X_{k-1},\left(X \wedge_{E} Y\right) X_{k}\right),
\end{aligned}
$$

where $\mathcal{E}$ is the endomorphism of $\chi(M)$ defined by $g(\mathcal{E} X, Y)=E(X, Y)$. Putting in the above formulas $T=R, T=S, T=C, T=K, T=Z$ or $T=P, E=g$ or $E=S$, we obtain the tensors: $R \cdot R, R \cdot S, R \cdot C, R \cdot K, C \cdot R, C \cdot S, C \cdot C, C \cdot K, Q(g, R)$, $Q(g, S), Q(g, C), Q(g, K), Q(S, R), Q(S, C), Q(g, K), S \cdot R, S \cdot C, S \cdot K, Z \cdot Z, Z \cdot R$, $R \cdot Z, Z \cdot S, P \cdot R, P \cdot P, R \cdot P, P \cdot S$, etc. The tensor $Q(E, T)$ is called the Tachibana tensor of the tensors $E$ and $T$, or the Tachibana tensor for short [34]. We note that (cf., [21, Section 4.4])

$$
\begin{aligned}
K \cdot K & =\left(R-\frac{r}{n(n-1)} G\right) \cdot\left(R-\frac{r}{n(n-1)} G\right) \\
& =R \cdot R-\frac{r}{n(n-1)} G \cdot R=R \cdot R-\frac{r}{n(n-1)} Q(g, R) .
\end{aligned}
$$

We also have (see, e.g., [38, Proposition 1])

$$
\begin{aligned}
C \cdot C & =\left(Z+\frac{r}{(n-1)(n-2)} G\right) \cdot\left(Z+\frac{r}{(n-1)(n-2)} G\right) \\
& =Z \cdot Z+\frac{r}{(n-1)(n-2)} G \cdot Z=Z \cdot Z+\frac{r}{(n-1)(n-2)} Q(g, Z) .
\end{aligned}
$$

If a semi-Riemannian manifold $(M, g), n \geq 3$, satisfies the condition $R \cdot R=0$, then it is called semisymmetric [9, 107-109].

As a proper generalization of semisymmetric manifold, the notion of pseudosymmetric manifolds arose during the study of semisymmetric totally umbilical submanifolds in manifolds admitting semisymmetric generalized curvature tensors [20]. A semi-Riemannian manifold $(M, g), n \geq 3$, is said to be pseudosymmetric by Deszcz $[23,35]$ (see also [13,113]) (resp. Ricci-pseudosymmetric [21,37]) if on $U_{R}=\{x \in M$ : $\left.\left(R-\frac{r}{n(n-1)} G\right)_{x} \neq 0\right\}$ (resp. on $\left.U_{S}\right), R \cdot R$ (resp. $R \cdot S$ ) and the Tachibana tensor $Q(g, R)$ (resp. $Q(g, S))$ are linearly dependent at every point of $M$. 
A semi-Riemannian manifold $(M, g), n \geq 4$, is said to be a manifold with pseudosymmetric Weyl conformal curvature tensor $[22,36]$ (see also $[13,26,46]$ ) if the tensor $C \cdot C$ and the Tachibana tensor $Q(g, C)$ are linearly dependent at every point of $M$, that is

$$
C \cdot C=L_{C} Q(g, C)
$$

on $U_{C}=\{x \in M: C \neq 0$ at $x\}$, where $L_{C}$ is some function on this set. We note that $U_{C} \cup U_{S}=U_{R}$ (see, e.g., [31]).

A semi-Riemannian manifold $(M, g), n \geq 3$, is said to be Ricci-generalized pseudosymmetric $[17,18]$ if at every point of $M$, the tensor $R \cdot R$ and the Tachibana tensor $Q(S, R)$ are linearly dependent. Hence $(M, g)$ is Ricci-generalized pseudosymmetric if and only if

$$
R \cdot R=L Q(S, R)
$$

holds on $U=\{x \in M: Q(S, R) \neq 0$ at $x\}$, where $L$ is some function on this set. An important subclass of Ricci-generalized pseudosymmetric manifolds is formed by the manifolds realizing the condition $[17,36]$

$$
R \cdot R=Q(S, R)
$$

The conditions of pseudosymmetry, Ricci-pseudosymmetry or any other conditions (obtained from various curvature tensors) of this kind are called pseudosymmetry type conditions.

A symmetric $(0,2)$-tensor $E$ on $M$ is called Riemann compatible or $R$-compatible [59,60] (see also [30]) if on $M$ we have

$$
R\left(\mathcal{E} X_{1}, X, X_{2}, X_{3}\right)+R\left(\mathcal{E} X_{2}, X, X_{3}, X_{1}\right)+R\left(\mathcal{E} X_{3}, X, X_{1}, X_{2}\right)=0
$$

for all $X, X_{1}, X_{2}, X_{3} \in \chi(M)$, where $\mathcal{E}$ is the endomorphism on $\chi(M)$ defined as

$$
g\left(\mathcal{E} X_{1}, X_{2}\right)=E\left(X_{1}, X_{2}\right) .
$$

Again a vector field $Y$ with associated 1-form $\Theta$, i.e., $g(Y, X)=\Theta(X)$ is called Riemann compatible or $R$-compatible [30,60] if

$$
\Theta\left(X_{1}\right) R\left(Y, X, X_{2}, X_{3}\right)+\Theta\left(X_{2}\right) R\left(Y, X, X_{3}, X_{1}\right)+\Theta\left(X_{3}\right) R\left(Y, X, X_{1}, X_{2}\right)=0,
$$

for all $X, X_{1}, X_{2}, X_{3} \in \chi(M)$. Similarly for other curvature tensors we have Weyl compatibility or $C$-compatibility [30,60,61], concircular compatibility, conharmonic compatibility etc. for both a vector and a $(0,2)$-tensor.

A semi-Riemannian manifold $(M, g)$ is said to be Roter type [24,27] (see also [32] and [41]) if its curvature tensor $R$ is expressed as the linear combination of $g \wedge g, g \wedge S$ and $S \wedge S$ i.e.,

$$
N R=N_{1} g \wedge g+N_{2} g \wedge S+N_{3} S \wedge S,
$$

where $N, N_{1}, N_{2}$ and $N_{3}$ are non-zero smooth functions on $M$.

Again a semi-Riemannian manifold $(M, g)$ is said to be generalized Roter type [83] if its curvature tensor $R$ is expressed as the linear combination of $S \wedge S, S \wedge S^{2}, g \wedge S$, 
$g \wedge S^{2}, g \wedge g$ and $S^{2} \wedge S^{2}$ i.e.,

$$
L R=L_{1} g \wedge g+L_{2} g \wedge S+L_{3} S \wedge S+L_{4} g \wedge S^{2}+L_{5} S \wedge S^{2}+L_{6} S^{2} \wedge S^{2},
$$

where $L$ and $L_{i}, 1 \leqslant i \leqslant 6$ are non-zero smooth functions on $M$. We note that any Roter type manifold is a generalized Roter type but not conversely. Very recently generalized Roter type manifolds were investigated in [31,97].

In [71, Theorem 1] it was stated that some 2-recurrent manifolds satisfy $R=\frac{1}{r} S \wedge S$. Curvature properties of pseudosymmetry type of manifolds satisfying the condition $R=\phi S \wedge S$, where $\phi$ is some function, were obtained in [54]. We also mention that in [42] manifolds satisfying some extensions of (2.4) were studied.

Again the curvature 2-forms $\Omega_{(R) l}^{m}[4,58]$ for the curvature tensor $R$ are given by

$$
\Omega_{(R) l}^{m}=R_{j k l}^{m} d x^{j} \wedge d x^{k}
$$

and Ricci 1-forms $\Lambda_{(S) l}$ are given by

$$
\Lambda_{(S) l}=S_{l m} d x^{m}
$$

where $\wedge$ indicates the exterior product. Recently, Mantica and Suh [62-65] determined the necessary and sufficient conditions for the recurrency of such curvature 2-forms and Ricci 1-forms. They showed that $\Omega_{(R) l}^{m}$ are recurrent (i.e., $\mathcal{D} \Omega_{(R) l}^{m}=A \wedge \Omega_{(R) l}^{m}, \mathcal{D}$ is the exterior derivative and $A$ is the associated 1-form) if and only if

$$
\begin{aligned}
& \nabla_{X_{1}} R\left(X_{2}, X_{3}, X, Y\right)+\nabla_{X_{2}} R\left(X_{3}, X_{1}, X, Y\right)+\nabla_{X_{3}} R\left(X_{1}, X_{2}, X, Y\right) \\
= & A\left(X_{1}\right) R\left(X_{2}, X_{3}, X, Y\right)+A\left(X_{2}\right) R\left(X_{3}, X_{1}, X, Y\right)+A\left(X_{3}\right) R\left(X_{1}, X_{2}, X, Y\right)
\end{aligned}
$$

and $\Lambda_{(S) l}$ are recurrent (i.e., $\left.\mathcal{D} \Lambda_{(S) l}=A \wedge \Lambda_{(S) l}\right)$ if and only if

$$
\nabla_{X_{1}} S\left(X_{2}, X\right)-\nabla_{X_{2}} S\left(X_{1}, X\right)=A\left(X_{1}\right) S\left(X_{2}, X\right)-A\left(X_{2}\right) S\left(X_{1}, X\right)
$$

for an 1 -form $A$. We can replace $R$ by some other curvature tensors such as conformal, concircular, conharmonic curvature tensor etc. and $S$ by any other symmetric $(0,2)$ tensors, and get the corresponding results.

\section{Curvature properties of the metric (1.1)}

Let $M$ be a non-empty open connected subset of $\mathbb{R}^{4}$ endowed with the metric given in (1.1). Then the non-zero components of the Christoffel symbols of second kind are given by:

$$
\Gamma_{11}^{1}=\Gamma_{12}^{2}=\Gamma_{13}^{3}=\frac{p}{1+2 p}=-\Gamma_{22}^{1}=-\Gamma_{33}^{1} .
$$

The non-zero components of curvature tensor and Ricci tensor are given by:

$$
\begin{gathered}
R_{1212}=R_{1313}=-\frac{p}{1+2 p}, \quad R_{2323}=-\frac{p^{2}}{1+2 p} \\
S_{11}=\frac{2 p}{(1+2 p)^{3}} g_{11}, \quad S_{22}=\frac{p(1+p)}{(1+2 p)^{3}} g_{22} \text { and } S_{33}=\frac{p(1+p)}{(1+2 p)^{3}} g_{33} .
\end{gathered}
$$


The scalar curvature of this metric is given by $r=\frac{2 p(2+p)}{(1+2 p)^{3}}$. Again, the non-zero local components of the Weyl conformal curvature tensor are given by:

$$
C_{1212}=C_{1313}=\frac{1}{2} C_{2323}=\frac{p(p-1)}{6(1+2 p)}, \quad C_{1414}=C_{2424}=\frac{1}{2} C_{3434}=\frac{p(p-1)}{6(1+2 p)^{2}} .
$$

Again, the non-zero components $R_{h i j k, l}$ and $S_{i j, l}$ of the covariant derivatives of curvature tensor and Ricci tensor are given by:

$$
\begin{gathered}
R_{1212,1}=R_{1313,1}=\frac{p(4 p-1)}{(1+2 p)^{2}}, \quad R_{1223,3}=-R_{1323,2}=-\frac{1}{2} R_{2323,1}=\frac{p^{2}(1-p)}{(1+2 p)^{2}}, \\
S_{11,1}=\frac{2 p(1-4 p)}{(1+2 p)^{3}}, \quad S_{12,2}=S_{13,3}=\frac{p^{2}(1-p)}{(1+2 p)^{3}}, \text { and } S_{22,1}=S_{33,1}=\frac{p\left(1-2 p-2 p^{2}\right)}{(1+2 p)^{2}} .
\end{gathered}
$$

We have made all the calculations by using a programme in Wolfram Mathematica.

\section{Conclusions}

Using the formulas given in Section 3 we can easily check curvature properties of the metric (1.1). Namely we have

Theorem 4.1. Let $\left(M^{4}, g\right)$ be a semi-Riemannian manifold equipped with the metric (1.1). Then $\left(M^{4}, g\right)$ satisfies the following:

(i) $C \cdot C=\frac{p(1-p)}{6(1+2 p)^{3}} Q(g, C)$,

(ii) $P \cdot R=\frac{2}{3} Q(S, R)$,

(iii) $C \cdot Z=\frac{p(1-p)}{6(1+2 p)^{3}} Q(g, Z)$,

(iv) $Z \cdot C=-\frac{p(1+p)}{2(1+2 p)^{3}} Q(g, C)$,

(v) $Z \cdot Z=-\frac{p(1+p)}{2(1+2 p)^{3}} Q(g, Z)$,

(vi) $R \cdot R=Q(S, R)$,

(vii) $R \cdot C+C \cdot R+\frac{p}{3(1+2 p)^{2}} Q(g, C)-Q(S, C)=0$,

(viii) $R \cdot Z+Z \cdot R+\frac{p(2+p)}{(1+2 p)^{3}} Q(g, R)-\frac{p}{(1+2 p)^{3}} Q(g, Z)-Q(S, Z)=0$,

(ix) $C \cdot K+K \cdot C+\frac{p(4+5 p)}{6(1+2 p)^{2}} Q(g, C)-Q(S, C)=0$,

(x) $K \cdot Z+Z \cdot K+\frac{p(2+p)}{(1+2 p)^{3}} Q(g, K)-\frac{p(4-p)}{6(1+2 p)^{3}} Q(g, Z)-Q(S, Z)=0$,

(xi) having no $R$-compatible vector but the $R$-compatible tensor and the $K$-compatible tensor are respectively of the form

$$
\left(\begin{array}{cccc}
a_{11} & a^{2} e^{-x^{1}} a_{21} & a^{2} e^{-x^{1}} a_{31} & a_{14} \\
a_{21} & a_{22} & a_{32} & a_{24} \\
a_{31} & a_{32} & a_{33} & a_{34} \\
0 & 0 & 0 & a_{44}
\end{array}\right) \text { and }\left(\begin{array}{cccc}
a_{11} & 0 & 0 & a_{14} \\
0 & a_{22} & a_{23} & 0 \\
0 & a_{23} & a_{33} & 0 \\
\frac{2 a^{2}+e^{x^{1}}}{-4 a^{2}+e^{x^{1}}} a_{14} & 0 & 0 & a_{44}
\end{array}\right)
$$


(xii) both the $C$-compatible and $Z$-compatible tensors are of the form

$$
\left(\begin{array}{cccc}
a_{11} & 0 & 0 & a_{14} \\
0 & a_{22} & a_{23} & 0 \\
0 & a_{23} & a_{33} & 0 \\
a_{14} & 0 & 0 & a_{44}
\end{array}\right)
$$

(xiii) Ricci 1-forms are recurrent with the 1-form of rercurrency

$$
A=\left(-\frac{p^{2}+3 p-1}{2 p^{2}+3 p+1}, 0,0,0\right),
$$

(xiv) curvature 2-forms for $C$, i.e., $\Omega_{(C) l}^{m}$ are recurrent with the 1-form of rercurrency

$$
A=\left(\frac{p^{2}+3 p-1}{2 p^{2}-p-1}, 0,0,0\right),
$$

(xv) Ricci tensor is Riemann compatible as well as $C, K$ and $Z$ compatible,

(xvi) does not satisfy the Roter type condition but it is a generalized Roter type manifold with

$$
\begin{aligned}
& L_{2}=-\frac{(3+p)(1+2 p)^{3}}{p(p+1)} L_{1}, \\
& L_{3}=\frac{2 p^{4}(1+p)^{4} L_{6}-p(1+2 p)^{9}+2(2+p)(1+2 p)^{12} L_{1}}{2 p^{2}(1+p)^{2}(1+2 p)^{6}}, \\
& L_{4}=\frac{(1+2 p)^{6}}{p^{2}(1+p)} L_{1}, \\
& L_{5}=\frac{p(1+2 p)^{9}-4 p^{4}(1+p)^{3} L_{6}-2(1+2 p)^{12} L_{1}}{2 p^{3}(1+p)^{2}(1+2 p)^{3}},
\end{aligned}
$$

where $L_{1}, L_{6}$ and $a_{i j}$ 's are arbitrary scalars,

(xvii) at all the points of the manifold at which $p \neq 1, \operatorname{rank}\left(S-\frac{p(1+p)}{(1+2 p)^{2}} g\right)=2$, and hence the manifold is 2-quasi-Einstein.

Remark 4.1. Let $\left(M^{4}, g\right)$ be a semi-Riemannian manifold equipped with the metric (1.1). Then $\left(M^{4}, g\right)$ does not satisfy any one of the following:

(i) quasi-Einstein,

(ii) Ricci recurrent,

(iii) weakly generalized recurrent,

(iv) hyper-generalized recurrent,

(v) quasi-generalized recurrent,

(vi) Codazzi type Ricci tensor,

(vii) cyclic Ricci parallel,

(viii) Ricci semisymmetric,

(ix) weakly symmetric,

(x) weakly Ricci symmetric, 


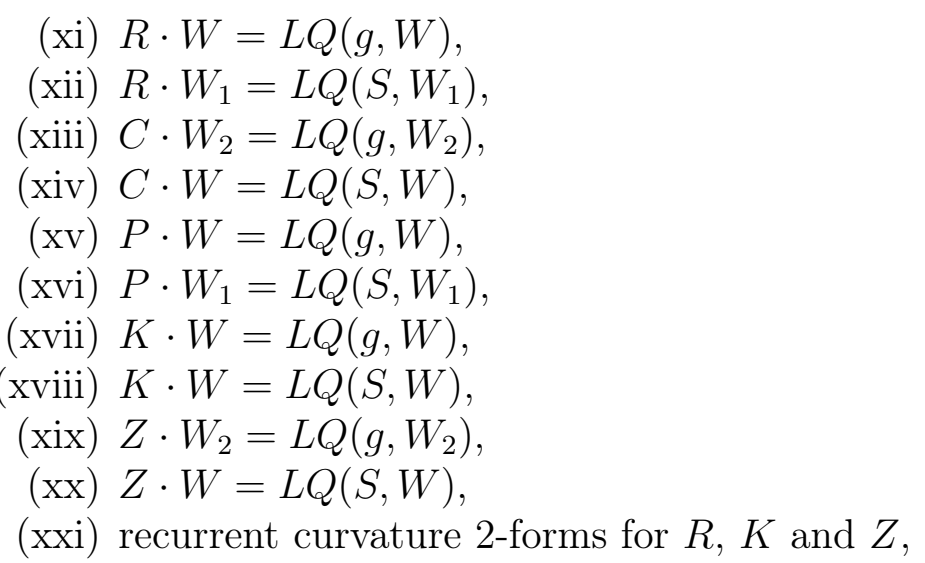

where $L$ is some smooth function, $W$ is any one of $R, C, P, K$ and $Z, W_{1}$ is any one of $C, P, K$ and $Z$ and $W_{2}$ is any one of $R, S, P, K$ and $Z$.

Remark 4.2. We note that the semi-Riemannian manifold $\left(M^{4}, g\right)$ equipped with the metric (1.1) is a product manifold of an 1-dimensional and a 3-dimensional manifold. Hence the condition presented in Theorem 4.1 (vi) is an immediate consequence of Corollary 3.2 of [18]. Again in [46, Theorem 2], Deszcz et al. showed that an warped product manifold with 1-dimensional base and 3-dimensional fiber satisfies $C \cdot C=L Q(g, C)$ if and only if the fiber is quasi-Einstein. Since the 3-dimensional part of $\left(M^{4}, g\right)$ is quasi-Einstein, the condition presented in Theorem 4.1 (i) is a consequence of Theorem 2 of [46] (see also Theorem 4.4 of [31]). Furthermore, it is obvious that the metric (1.1) can also be considered as an warped product manifold with 2-dimensional base manifold and 2-dimensional fiber. Some curvature properties of such manifolds are presented in Theorem 6.2 and Theorem 7.1 of [31].

Again, if we consider the signature of the metric (1.1) as semi-Riemannian, and in particular, Lorentzian, given by

$$
d s^{2}=g_{i j} d x^{i} d x^{j}=(1+2 p)\left[\left(d x^{1}\right)^{2} \pm\left(d x^{2}\right)^{2} \pm\left(d x^{3}\right)^{2}\right] \pm\left(d x^{4}\right)^{2},
$$

$i, j=1,2,3,4$, then it can be easily shown that the obtained results remain unchanged. Again if we consider the metric as:

$$
d s^{2}=g_{i j} d x^{i} d x^{j}=(1+2 p)\left[\left(d x^{1}\right)^{2}+\left(d x^{2}\right)^{2}+\left(d x^{4}\right)^{2}\right]+\left(d x^{3}\right)^{2}
$$

and

$$
d s^{2}=g_{i j} d x^{i} d x^{j}=(1+2 p)\left[\left(d x^{1}\right)^{2}+\left(d x^{3}\right)^{2}+\left(d x^{4}\right)^{2}\right]+\left(d x^{2}\right)^{2},
$$

$i, j=1,2,3,4$, then the obtained results also remain unchanged. It may be mentioned that if the signature of the metrics (4.1) and (4.2) are considered as semi-Riemannian, and in particular, Lorentzian, then also the results will be the same.

Again, if we consider the signature of the metric (1.1) as Lorentzian given by

$$
d s^{2}=g_{i j} d x^{i} d x^{j}=(1+2 p)\left[-\left(d x^{1}\right)^{2}+\left(d x^{2}\right)^{2}+\left(d x^{3}\right)^{2}\right]+\left(d x^{4}\right)^{2},
$$


$i, j=1,2,3,4$, then it is easy to check that the metrics (4.3) gives the same results as metric (1.1) but only $L_{1}, L_{3}, L_{4}$ and $L_{5}$ are changed by negative sign.

Now another kind of metric which is nearly similar as metric (1.1) is given below a conformally flat metric [89, 100]:

$$
d s^{2}=g_{i j} d x^{i} d x^{j}=(1+2 p)\left[\left(d x^{1}\right)^{2}+\left(d x^{2}\right)^{2}+\left(d x^{3}\right)^{2}+\left(d x^{4}\right)^{2}\right], \quad i, j=1,2,3,4,
$$

where $p=\frac{e^{x^{1}}}{\rho^{2}}$ and $\rho$ is a non-zero constant; $x^{1}, \ldots, x^{4}$ are the standard coordinates of $\mathbb{R}^{4}$. Let $M$ be a non-empty open connected subset of $\mathbb{R}^{4}$ endowed with the metric (4.4). Later the same metric is used in [16].

Then the non-zero components of the Christoffel symbols of second kind are given by:

$$
\Gamma_{11}^{1}=\Gamma_{12}^{2}=\Gamma_{13}^{3}=\Gamma_{14}^{4}=\frac{p}{1+2 p}=-\Gamma_{22}^{1}=-\Gamma_{33}^{1}=-\Gamma_{44}^{1} .
$$

The non-zero components of curvature tensor and Ricci tensor are given by:

$$
\left\{\begin{array}{l}
R_{1212}=R_{1313}=R_{1414}=-\frac{p}{1+2 p}, \quad R_{2323}=R_{2424}=R_{3434}=-\frac{p^{2}}{1+2 p} \\
S_{11}=\frac{3 p}{(1+2 p)^{3}} g_{11}, \quad S_{22}=\frac{p}{(1+2 p)^{2}} g_{22}, \quad S_{33}=\frac{p}{(1+2 p)^{2}} g_{33}, \quad S_{44}=\frac{p}{(1+2 p)^{2}} g_{44}
\end{array}\right.
$$

The scalar curvature of this metric is given by $r=\frac{6 p(1+p)}{(1+2 p)^{3}}$. Again the non-zero components $R_{h i j k, l}$ and $S_{i j, l}$ of the covariant derivatives of curvature tensor and Ricci tensor are given by:

$$
\begin{aligned}
& \left\{\begin{array}{l}
R_{1212,1}=R_{1313,1}=R_{1414,1}=\frac{p(4 p-1)}{(1+2 p)^{2}}, R_{2323,1}=R_{2424,1}=R_{3434,1}=\frac{2 p^{2}(p-1)}{(1+2 p)^{2}} \\
R_{1223,3}=-R_{1323,2}=R_{1224,4}=R_{1334,4}=R_{1424,2}=R_{1434,3}=\frac{p^{2}(1-p)}{(1+2 p)^{2}}
\end{array}\right. \\
& \left\{\begin{array}{l}
S_{11,1}=\frac{3 p(1-4 p)}{(1+2 p)^{3}}, \quad S_{22,1}=S_{33,1}=S_{44,1}=\frac{p(1-2 p)}{(1+2 p)^{2}} \\
S_{12,2}=S_{13,3}=S_{14,4}=\frac{2 p^{2}(1-p)}{(1+2 p)^{3}}
\end{array}\right.
\end{aligned}
$$

Using the formulas (4.4)-(4.7) we can easily check curvature properties of the metric (4.4). Namely we have

Theorem 4.2. Let $\left(M^{4}, g\right)$ be a semi-Riemannian manifold equipped with the metric (4.4). Then $\left(M^{4}, g\right)$ satisfies the following:

(i) quasi-Einstein,

(ii) conformally flat,

(iii) conharmonicly recurrent with the 1 -form of recurrency $A=\left(-\frac{2 p^{2}+2 p-1}{2 p^{2}+3 p+1}, 0,0,0\right)$,

(iv) $R \cdot R=Q(S, R)$,

(v) $Z \cdot R=Z \cdot K=-\frac{1}{2} Q(S, Z)$,

(vi) $R \cdot W=\frac{p}{(1+2 p)^{3}} Q(g, W)$,

(vii) $K \cdot W=\frac{p(1-p)}{2(1+2 p)^{3}} Q(g, W)$,

(viii) $Z \cdot W=-\frac{p(1+p)}{(1+2 p)^{3}} Q(g, W)$,

(ix) $P \cdot R=\frac{2}{3} Q(S, R)$, 


$$
\begin{aligned}
& \text { (x) } P \cdot Z=-\frac{1}{3} Q(S, Z), \\
& \text { (xi) } P \cdot R=\frac{2 p}{3(1+2 p)^{3}} Q(g, R), \\
& \text { (xii) } P \cdot S=\frac{p}{(1+2 p)^{3}} Q(g, S), \\
& \text { (xiii) } R \cdot K=\frac{1}{2+p} Q(S, K), \\
& \text { (xiv) } P \cdot K=\frac{p(1-p)}{3(1+2 p)^{3}} Q(g, K), \\
& \text { (xv) } P \cdot K=\frac{1-p}{3(2+p)} Q(S, K), \\
& \text { (xvi) } P \cdot Z=-\frac{1}{3} Q(S, Z), \\
& \text { (xvii) } K \cdot R=\frac{1}{2}(1-p) Q(S, R), \\
& \text { (xviii) } K \cdot K=\frac{1-p}{2(2+p)} Q(S, K), \\
& \text { (xix) } Z \cdot R=-(1+p) Q(S, R), \\
& \text { (xx) } Z \cdot K=-\frac{1+p}{2+p} Q(S, K), \\
& \text { (xxi) } Q(S, R)=\frac{1}{2(1+p)} Q(S, Z), \\
& \text { (xxii) } Q(S, K)=\frac{2+p}{2(1+p)} Q(S, Z), \\
& \text { (xxiii) } R \cdot K-Q(S, K)=-\frac{p(1+p)}{(1+2 p)^{3}} Q(g, R)=-(1+p) Q(S, R), \\
& \text { (xxiv) } R \cdot K-Q(g, K)=-\frac{1+5 p+12 p^{2}+8 p^{3}}{p} Q(S, Z), \\
& \text { (xxv) } T h e R \text {-compatible tensor and the } K \text {-compatible tensor are respectively of the }
\end{aligned}
$$
form

$$
\left(\begin{array}{cccc}
a_{11} & a^{2} e^{-x^{1}} a_{21} & a^{2} e^{2-x^{1}} a_{31} & a^{2} e^{-x^{1}} a_{41} \\
a_{21} & a_{22} & a_{32} & a_{42} \\
a_{31} & a_{32} & a_{33} & a_{43} \\
a_{41} & a_{42} & a_{43} & a_{44}
\end{array}\right) \text { and }\left(\begin{array}{cccc}
a_{11} & -a_{21} & -a_{31} & -a_{41} \\
a_{21} & a_{22} & a_{32} & a_{42} \\
a_{31} & a_{32} & a_{33} & a_{43} \\
a_{41} & a_{42} & a_{43} & a_{44}
\end{array}\right) \text {, }
$$

(xxvi) any symmetric $(0,2)$ tensor is $Z$-compatible,

(xxvii) Ricci tensor is $R, K$ and also $Z$-compatible,

(xxviii) Ricci 1-forms are recurrent with the 1-form of recurrency $\left(\frac{1-2 p-2 p^{2}}{(1+2 p)^{2}}, 0,0,0\right)$, (xxix) curvature 2-forms for $K$ are recurrent with the 1-form of recurrency

$$
\left(\frac{2 p^{2}+2 p-1}{2 p^{2}-p-1}, 0,0,0\right),
$$

(xxx) curvature 2-forms for $Z$ are recurrent with the 1-form of recurrency

$$
\left(-\frac{2 p^{2}+2 p-1}{2 p^{2}+3 p+1}, 0,0,0\right)
$$

(xxxi) Roter type manifold with

$$
N_{2}=-\frac{2(1+2 p)^{2}}{p} N_{1}-\frac{1}{2(1+2 p)}, \quad N_{3}=-\frac{(1+2 p)\left(p(1+p)+2 N_{1}(1+2 p)^{3}\right)}{2 p^{2}},
$$

where $W$ is any one of $R, S, P, K$ and $N_{1}, a_{i j}$ 's are any scalar.

Remark 4.3. Let $\left(M^{4}, g\right)$ be a semi-Riemannian manifold equipped with the metric (4.4). Then $\left(M^{4}, g\right)$ does not satisfy any one of the following: (i) Einstein, (ii) Ricci 
recurrent, (iii) weakly generalized recurrent, (iv) hyper- generalized recurrent, (v) quasi-generalized recurrent, (vi) Codazzi type Ricci tensor, (vii) cyclic Ricci parallel, (viii) Ricci semisymmetric, (ix) weakly symmetric, (x) weakly Ricci symmetric, (xi) $W \cdot P=L Q(S, P)$, where $L$ is any smooth function, $W$ is any one of $R, K$ and $Z$.

Acknowledgements. The authors wish to express their sincere thanks and gratitude to the referees for their valuable suggestions towards the improvement of the paper. The work was started while the first author visited to Chuo University, Japan as a Guest Professor in 2012.

\section{REFERENCES}

[1] B. E. Abdalla and F. Dillen, A Ricci-semi-symmetric hypersurface of Euclidean space which is not semi-symmetric, Proc. Amer. Math. Soc. 130 (2002), 1805-1808.

[2] T. Adati and T. Miyazawa, On Riemannian space with recurrent conformal curvature, Tensor (N.S.) 18 (1967), 348-354.

[3] T. Adati and T. Miyazawa, On projective transformations of projective recurrent spaces, Tensor (N.S.) 31 (1977), 49-54.

[4] A. L. Besse, Einstein Manifolds, Springer-Verlag, Berlin, 1987.

[5] N. Bokan, Prof. Dr Mileva Prvanović - her contribution to differential geometry, Kragujevac J. Math. 25 (2003), 111-125.

[6] É. Cartan, La déformation des hypersurfaces dans l'espace conforme réel a $n \geq 5$ dimensions, Bull. Soc. Math. France 45 (1917), 57-121.

[7] É. Cartan, Sur une classe remarquable d'espaces de Riemann I, Bull. de la Soc. Math. de France 54 (1926), 214-216.

[8] É. Cartan, Sur une classe remarquable d'espaces de Riemann II, Bull. de la Soc. Math. de France 55 (1927), 114-134.

[9] É. Cartan, Lecons sur la geometrie des espaces de Riemann, 2nd ed., Gauthier-Villars, Chapman \& Hall, Paris, 1946.

[10] M. C. Chaki, On pseudosymmetric manifolds, An. Ştiinţ. Univ. Al. I. Cuza Iaşi. Mat. (N.S.) 33 (1987), 53-58.

[11] M. C. Chaki, On pseudo Ricci symmetric manifolds, Bulgarian J. Phys. 15 (1988), 526-531.

[12] M. C. Chaki and U. C. De, On pseudo symmetric manifolds, Acta Math. Hungarica 35 (1989), $185-190$.

[13] B.-Y. Chen, Geometric and topological obstructions to various immersions in submanifold theory and some related open problems, Kragujevac J. Math. 39 (2015), 93-109.

[14] J. Chojnacka-Dulas, R. Deszcz, M. Głogowska and M. Prvanović, On warped product manifolds satisfying some curvature conditions, J. Geom. Phys. 74 (2013), 328-341.

[15] A. De, C. Özgür and U. C. De, On conformally flat almost pseudo-Ricci symmetric spacetimes, Int. J. Theo. Phys. 51 (2012), 2878-2887.

[16] U. C. De and S. Mallick, On almost pseudo concircularly symmetric manifolds, J. Math. Comp. Sci. 4 (2012), 317-330.

[17] F. Defever and R. Deszcz, On semi-Riemannian manifolds satisfying the condition $R \cdot R=$ $Q(S, R)$, in: Geometry and Topology of Submanifolds III, World Sci., 1991, pp. 108-130.

[18] F. Defever and R. Deszcz, On warped product manifolds satisfying a certain curvature condition, Atti. Acad. Peloritana Pericolanti Cl. Sci. Fis. Mat. Natur. 69 (1991), 213-236.

[19] F. Defever, R. Deszcz, M. Hotloś, M. Kucharski and Z. Sentürk, Generalisations of RobertsonWalker spaces, Ann. Univ. Sci. Budapest. Eötvös Sect. Math. 43 (2000), 13-24. 
[20] R. Deszcz, Notes on totally umbilical submanifolds, in: J. M. Morvan and L. Verstraelen (Eds.), Geometry and Topology of Submanifolds, vol. I, World Sci. Publ., Singapore, Luminy, 1989, pp. 89-97.

[21] R. Deszcz, On Ricci-pseudosymmetric warped products, Demonstratio Math. 22 (1989), 10531065.

[22] R. Deszcz, On four-dimensional warped product manifolds satisfying certain pseudosymmetry curvature conditions, Colloq. Math. 62 (1991), 103-120.

[23] R. Deszcz, On pseudosymmetric spaces, Bull. Belgian Math. Soc., Series A 44 (1992), 1-34.

[24] R. Deszcz, some akivis-goldberg type metrics, Publ. Inst. Math. (Beograd) (N.S.) 74 (2003), 71-83.

[25] R. Deszcz, F. Dillen, L. Verstraelen and L. Vrancken, Quasi-Einstein totally real submanifolds of the nearly Kähler 6-sphere, Tohoku Math. J. 51 (1999), 461-478.

[26] R. Deszcz, M. Głogowska, H. Hashiguchi, M. Hotloś and M. Yawata, On semi-Riemannian manifolds satisfying some conformally invariant curvature condition, Colloq. Math. 131 (2013), 149-170.

[27] R. Deszcz, M. Głogowska, M. Hotloś and K. Sawicz, A survey on generalized Einstein metric conditions, in: A. R. M. Plaue and M. Scherfner (Eds.), Advances in Lorentzian Geometry: Proceedings of the Lorentzian Geometry Conference in Berlin, AMS/IP Stud. Adv. Math, vol. 49, AMS, Berlin, 2011, pp. 27-46.

[28] R. Deszcz, M. Głogowska, M. Hotloś and Z. Sentürk, On certain quasi-Einstein semi-symmetric hypersurfaces, Ann. Univ. Sci. Budapest. Eötvös Sect. Math. 41 (1998), 151-164.

[29] R. Deszcz, M. Głogowska, M. Hotloś and G. Zafindratafa, Hypersurfaces in space forms satisfying some curvature conditions, J. Geom. Phys. 99 (2016), 218-231.

[30] R. Deszcz, M. Głogowska, J. Jełowicki, M. Petrović-Torgašev and G. Zafindratafa, On Riemann and Weyl compatible tensors, Publ. Inst. Math. (Beograd) (N.S.) 94 (2013), 111-124.

[31] R. Deszcz, M. Głogowska, J. Jełowicki and G. Zafindratafa, Curvature properties of some class of warped product manifolds, Int. J. Geom. Method Mod. Phys. 13 (2016), 1550135 (36 pages).

[32] R. Deszcz, M. Głogowska, M. Petrović-Torgašev and L. Verstraelen, On the Roter type of Chen ideal submanifolds, Results in Math. 59 (2011), 401-413.

[33] R. Deszcz, M. Głogowska, M. Petrović-Torgašev and L. Verstraelen, Curvature properties of some class of minimal hypersurfaces in Euclidean spaces, Filomat 29 (2015), 479-492.

[34] R. Deszcz, M. Głogowska, M. Plaue, K. Sawicz and M. Scherfner, On hypersurfaces in space forms satisfying particular curvature conditions of Tachibana type, Kragujevac J. Math. 35 (2011), 223-247.

[35] R. Deszcz and W. Grycak, On some class of warped product manifolds, Bull. Inst. Math. Acad. Sinica 15 (1987), 311-322.

[36] R. Deszcz and W. Grycak, On manifolds satisfying some curvature conditions, Colloq. Math. 57 (1989), 89-92.

[37] R. Deszcz and M. Hotloś, Remarks on Riemannian manifolds satisfying certain curvature condition imposed on the Ricci tensor, Prace Nauk. Pol. Szczec. 11 (1989), 23-34.

[38] R. Deszcz, M. Hotloś, J. Jełowicki, H. Kundu and A. A. Shaikh, Curvature properties of Gödel metric, Int. J. Geom. Method Mod. Phy. 11 (2014), Article ID 1450025.

[39] R. Deszcz, M. Hotloś and Z. Sentürk, On curvature properties of quasi-Einstein hypersurfaces in semi-Euclidean spaces, Soochow J. Math. 27 (2001), 375-390.

[40] R. Deszcz, M. Hotloś and Z. Sentürk, Quasi-Einstein hypersurfaces in semi-Riemannian space forms, Colloq. Math. 81 (2001), 81-97.

[41] R. Deszcz and D. Kowalczyk, On some class of pseudosymmetric warped products, Colloq. Math. 97 (2003), 7-22.

[42] R. Deszcz and M. Prvanović, Roter type equations for a class of anti-Kähler manifolds, Ann. Univ. Sci. Budapest. Eötvös Sect. Math. 52 (2009), 103-121. 
[43] R. Deszcz, P. Verheyen and L. Verstraelen, On some generalized Einstein metric conditions, Publ. Inst. Math. (Beograd) (N.S.) 60 (1996), 108-120.

[44] R. Deszcz and L. Verstraelen, Hypersurfaces of semi-Riemannian conformally flat manifolds, in: Geometry and Topology of Submanifolds, III, World Sci., River Edge, NJ, 1991, pp. 131-147.

[45] R. Deszcz, L. Verstraelen and S. Yaprak, Pseudosymmetric hypersurfaces in 4-dimensional spaces of constant curvature, Bull. Inst. Math. Acad. Sinica 22 (1994), 167-179.

[46] R. Deszcz, L. Verstraelen and Ș. Yaprak, Warped products realizing a certain condition of pseudosymmetry type imposed on the Weyl curvature tensor, Chinese J. Math. 22 (1994), 139-157.

[47] R. S. D. Dubey, Generalized recurrent spaces, Indian J. Pure Appl. Math. 10 (1979), 1508-1513.

[48] D. Ferus, A remark on Codazzi tensors in constant curvature spaces, in: Global differential geometry and global analysis, Springer, 1981, pp. 257-257.

[49] M. Głogowska, On quasi-Einstein Cartan type hypersurfaces, J. Geom. Phys. 58 (2008), 599614.

[50] A. Gray, Einstein-like manifolds which are not Einstein, Geom. Dedicata 7 (1978), 259-280.

[51] J. B. Griffiths and J. Podolský, Exact Space-Times in Einstein's General Relativity, Cambridge Univ. Press, New York, 1996.

[52] S. K. Hui, Y. Matsuyama and A. A. Shaikh, On decomposable weakly conformally symmetric manifolds, Acta Math. Hungarica 128 (2010), 82-95.

[53] Y. Ishii, On conharmonic transformations, Tensor (N.S.) 7 (1957), 73-80.

[54] D. Kowalczyk, On some class of semisymmetric manifolds, Soochow J. Math. 27 (2001), 445-462.

[55] D. Kowalczyk, On the Reissner-Nordström-de sitter type spacetimes, Tsukuba J. Math. 30 (2006), 263-281.

[56] G. I. Kruchkovich, On spaces of V.F. Kagan, Appendix to the book: V.F. Kagan, Subprojective spaces, Moscow (1961), 163-198.

[57] A. Lichnerowicz, Courbure, nombres de Betti, et espaces symmetriques, in: Proc. of the Int. Cong. of Math., vol. 2, 1952, pp. 216-223.

[58] D. Lovelock and H. Rund, Tensor, Differential Forms and Variational Principle, Courier Dover Publications, 1989.

[59] C. A. Mantica and L. G. Molinari, Extended Derdzinski-Shen theorem for curvature tensors, Colloq. Math. 128 (2012), 1-6.

[60] C. A. Mantica and L. G. Molinari, Riemann compatible tensors, Colloq. Math. 128 (2012), $197-210$.

[61] C. A. Mantica and L. G. Molinari, Weyl compatible tensors, Int. J. Geom. Methods Mod. Phys. 11 (2014), 1450070 (15 pp).

[62] C. A. Mantica and Y. J. Suh, The closedness of some generalized curvature 2-forms on a Riemannian manifold I, Publ. Math. Debrecen 81 (2012), 313-326.

[63] C. A. Mantica and Y. J. Suh, Recurrent Z-forms on Riemannian and Kaeheler manifolds, Int. J. Geom. Meth. Mod. Phys. 9 (2012), Article ID 1250059.

[64] C. A. Mantica and Y. J. Suh, The closedness of some generalized curvature 2-forms on a Riemannian manifold II, Publ. Math. Debrecen 82 (2013), 163-182.

[65] C. A. Mantica and Y. J. Suh, Recurrent conformal 2-forms on pseudo-Riemannian manifolds, Int. J. Geom. Meth. Mod. Phy. 11 (2014), Article ID 1450056.

[66] Y. Matsuyama, Complete hypersurfaces with $R \cdot S=0$ in $E^{n+1}$, Proc. Amer. Math. Soc. 88 (1983), 119-123.

[67] S. M. Minčić and L. S. Velimirović, Academician Mileva Prvanović - the first doctor of geometrical sciences in Serbia, Filomat 29 (2015), 375-380.

[68] V. A. Mirzoyan, Structure theorems for Riemannian Ric-semisymmetric spaces (Russian), Izv. Vyssh. Uchebn. Zaved. Mat. 6 (1992), 80-89. 
[69] M. Okumura, Some remarks on space with a certain contact structure, Tohoku Math. J., 2nd Ser. 14 (1962), 135-145.

[70] E. M. Patterson, Some theorems on Ricci recurrent spaces, J. London Math. Soc. 27 (1952), 287-295.

[71] W. Roter, A note on second order recurrent spaces, Bull. Acad. Polon. Sci., Sér. Sci. Math., Astr. et Phys. 10 (1964), 621-626.

[72] W. Roter, Some remarks on second order recurrent spaces, Bull. Acad. Polon. Sci., Sér. Sci. Math., Astr. et Phys. 12 (1964), 207-211.

[73] H. Ruse, On simply harmonic spaces, J. London Math. Soc. 21 (1946), 243-247.

[74] H. Ruse, On simply harmonic 'kappa spaces' of four dimensions, Proc. London Math. Soc. 50 (1949), 317-329.

[75] H. S. Ruse, Three dimensional spaces of recurrent curvature, Proc. London Math. Soc. 50 (1949), 438-446.

[76] P. J. Ryan, Hypersurfaces with parallel Ricci tensor, Osaka J. Math. 8 (1971), 251-259.

[77] J. A. Schouten, Über die konforme abbildung n-dimensionaler mannigfaltigkeiten mit quadratischer massbestimmung auf eine mannigfaltigkeit mit euklidischer massbestimmung, Math. Z. 11 (1921), 58-88.

[78] A. Selberg, Harmonic analysis and discontinuous groups in weakly symmetric Riemannian spaces with applications to dirichlet series, Indian J. of Math. 20 (1956), 47-87.

[79] R. N. Sen and M. C. Chaki, On curvature restrictions of a certain kind of conformally flat Riemannian space of class one, Proc. Nat. Inst. Sci. 33 (1967), 100-102.

[80] A. A. Shaikh, F. R. Al-Solamy and I. Roy, On the existence of a new class of semi-Riemannian manifolds, Math. Sci. 7 (2013), 1-13.

[81] A. A. Shaikh and K. K. Baishya, On weakly quasi-conformally symmetric manifolds, Soochow J. of Math. 31 (2005), 581-595.

[82] A. A. Shaikh and T. Q. Binh, On some class of Riemannian manifolds, Bull. Transilvania Univ. 15 (2008), 351-362.

[83] A. A. Shaikh, R. Deszcz, M. Hotloś, J. Jełowicki and H. Kundu, On pseudosymmetric manifolds, Publ. Math. Debrecen 86 (120158), 433-456.

[84] A. A. Shaikh and S. K. Hui, On decomposable weakly conharmonically symmetric manifolds, Lobachevski J. Math. 29 (2008), 206-215.

[85] A. A. Shaikh and S. K. Hui, On weakly conharmonically symmetric manifolds, Tensor (N.S.) 70 (2008), 119-134.

[86] A. A. Shaikh and S. K. Hui, On weakly concircular symmetric manifolds, Sci. Annals of Al. I. Cuza, Din Iasi LV (2009), 167-186.

[87] A. A. Shaikh and S. K. Hui, On weakly projective symmetric manifolds, Acta Math. Academiae Paedagogicae Nyiregyhaziensis 25 (2009), 247-269.

[88] A. A. Shaikh and S. K. Hui, On decomposable quasi-Einstein spaces, Math. Reports 13 (2011), 89-94.

[89] A. A. Shaikh and S. K. Jana, On weakly cyclic Ricci symmetric manifolds, Ann. Polon. Math. 89 (2006), 273-288.

[90] A. A. Shaikh and S. K. Jana, On quasi-conformally flat weakly Ricci symmetric manifolds, Acta Math. Hung. 115 (2007), 197-214.

[91] A. A. Shaikh and S. K. Jana, On weakly quasi-conformally symmetric manifolds, SUT J. Math. 41 (2007), 61-83.

[92] A. A. Shaikh and S. K. Jana, On weakly symmetric manifolds, Publ. Math. Debrecen 71 (2007), $27-41$.

[93] A. A. Shaikh, S. K. Jana and S. Eyasmin, On weakly $W_{2}$-symmetric manifolds, Sarajevo J. Math. 15 (2007), 1-19. 
[94] A. A. Shaikh, Y. H. Kim and S. K. Hui, On Lorentzian quasi-Einstein manifolds, J. Korean Math. Soc. 81 (2012), 487-505.

[95] A. A. Shaikh and H. Kundu, On weakly symmetric and weakly Ricci symmetric warped product manifolds, Publ. Math. Debrecen 81 (2012), 487-505.

[96] A. A. Shaikh and H. Kundu, On equivalency of various geometric structures, J. Geom. 105 (2014), 139-165.

[97] A. A. Shaikh and H. Kundu, On warped product generalized Roter type manifolds, Balkan J. Geom. Appl. 22 (2016), 82-95.

[98] A. A. Shaikh, H. Kundu and M. S. Ali, On warped product super generalized recurrent manifolds, An. Ştiinţ. Univ. Al. I. Cuza Iaşi. Mat. (N.S.), (in press).

[99] A. A. Shaikh, Y. Matsuyama, S. K. Jana and S. Eyasmin, On the existence of weakly Ricci symmetric manifolds admitting semi-symmetric metric connection, Tensor (N.S.) 70 (2008), 95-106.

[100] A. A. Shaikh and A. Patra, On a generalized class of recurrent manifolds, Arch. Math. (Brno) 46 (2010), 71-78.

[101] A. A. Shaikh and I. Roy, On quasi generalized recurrent manifolds, Math. Pannonica 20 (2010), 251-263.

[102] A. A. Shaikh and I. Roy, On weakly generalized recurrent manifolds, Ann. Univ. Sci. Budapest. Eötvös Sect. Math. 54 (2011), 33-45.

[103] A. A. Shaikh, I. Roy and H. Kundu, On some generalized recurrent manifolds, Bull. Iranian Math. Soc., (in press).

[104] A. A. Shaikh, M. Shahid and S. K. Hui, On weakly conformally symmetric manifolds, Math. Vesnik 60 (2008), 269-284.

[105] A. A. Shaikh, D. W. Yoon and S. K. Hui, On quasi-Einstein spacetimes, Tsukuba J. Math. 33 (2009), 305-326.

[106] U. Simon, Codazzi tensors, Glob. Diff. Geom. and Glob. Ann., Lecture notes 838 (1981), 289-296.

[107] Z. I. Szabó, Structure theorems on Riemannian spaces satisfying $R(X, Y) \cdot R=0$, I, the local version, J. Diff. Geom. 17 (1982), 531-582.

[108] Z. I. Szabó, Classification and construction of complete hypersurfaces satisfying $R(X, Y) \cdot R=0$, Acta Sci. Math. 47 (1984), 321-348.

[109] Z. I. Szabó, Structure theorems on Riemannian spaces satisfying $R(X, Y) \cdot R=0$, II, the global version, Geom. Dedicata 19 (1985), 65-108.

[110] L. Támassy and T. Q. Binh, On weakly symmetric and weakly projective symmetric Riemannian manifolds, Coll. Math. Soc. J. Bolyai 50 (1989), 663-67.

[111] L. Támassy and T. Q. Binh, On weak symmetrics of Einstein and Sasakian manifolds, Tensor (N.S.) 53 (1993), 140-148.

[112] S. Tanno, Hypersurfaces satisfying a certain condition on the Ricci tensor, Tohoku Math. J. 21 (1969), 297-303.

[113] L. Verstraelen, A concise mini history of geometry, Kragujevac J. Math. 38 (2014), 5-14.

[114] A. G. Walker, On Ruse's spaces of recurrent curvature, Proc. London Math. Soc. 52 (1950), 36-64.

[115] K. Yano and M. Kon, Structures on Manifolds, World Sci. Publ., Singapore, 1989. 
${ }^{1}$ Department of Mathematics,

UNIVERSITY OF BURDWAN,

Burdwan, Golapbag, Burdwan-713104

West Bengal, IndiA

E-mail address: aask2003@yahoo.co.in, aashaikh@math.buruniv.ac.in

${ }^{2}$ Department of Mathematics,

Chuo University,

KASUGA, BUNKYO-KU

TOKYO 112-8551, JAPAN

E-mail address: matuyama@math.chuo-u.ac.jp 